\title{
A carrier sense multiple access protocol with power backoff $(\mathrm{CSMA} / \mathrm{PB})$ 光
}

\author{
Charles J. Colbourn ${ }^{\text {a }}$, Minghao Cui ${ }^{\mathrm{a}, *}$, Errol L. Lloyd ${ }^{\mathrm{b}}$, Violet R. Syrotiuk ${ }^{\mathrm{a}}$ \\ a Department of Computer Science and Engineering, Arizona State University, Tempe, AZ 85287-8809, USA \\ b Department of Computer and Information Sciences, University of Delaware, Newark, DE 19716, USA
}

Available online 15 March 2007

\begin{abstract}
In this paper, we exploit space as a new dimension in collision resolution for a carrier sense multiple access (CSMA) protocol. Most contention-based medium access control protocols resolve collisions by backing off in time. We introduce power backoff (PB), the use of transmission power control to resolve collisions by backing off in space, and incorporate it into a CSMA protocol as CSMA/PB. Through analysis and simulation, we show that collision resolution using power backoff can be remarkably successful. Simulation results show that CSMA/PB outperforms IEEE 802.11 in both static and mobile ad hoc network scenarios. CSMA/PB improves end-to-end throughput and uses less energy. The resulting gains in throughput per unit energy can be substantial.
\end{abstract}

(C) 2007 Elsevier B.V. All rights reserved.

Keywords: Carrier sense multiple access; Collision resolution; Spatial backoff

\section{Introduction}

Recently, Gomez and Campbell $[1,2]$ investigated the impact of variable-range power control on the physical network connectivity, the network capacity, and the energy savings of wireless multi-hop networks. Inspired by their challenge to develop protocols that exploit power control we propose a

\footnotetext{
Prepared through collaborative participation in the Communications and Networks Consortium sponsored by the US Army Research Laboratory under the Collaborative Technology Alliance Program, Cooperative Agreement DAAD19-01-2-0011. The US Government is authorized to reproduce and distribute reprints for Government purposes not withstanding any copyright notation thereon.

Corresponding author.

E-mail address: Minghao.Cui@asu.edu (M. Cui).
}

medium access control (MAC) protocol for mobile ad hoc networks adding the dimension of space to collision resolution.

Traditional contention-based MAC protocols resolve collisions by backing off in time. A transmitter increases its contention window size when the channel is already in use or the previous transmission attempt fails. The transmitter then waits for a period related to the window size in the hope that contention is reduced. In contrast, we consider a transmitter that backs off in space-it reduces its transmission power. This is analogous to increasing the size of the contention window in temporal backoff; in a smaller transmission range, the interference and contention are likely to be reduced.

To see why backing off in space may be as (or more) effective than backing off in time, consider a 
network in which the nodes are uniformly distributed throughout some geographic area. If a node has a transmission range of $r$, then the physical area of the interference is proportional to $r^{2}$. If the network traffic is also uniformly distributed among nodes, then the magnitude of the contention at each node is also proportional to $r^{2}$. Since the contention increases in proportion to the square of the transmission range, the reduction of the transmission range by half, for example, results in a four fold decrease in the contention. Consider a path from a source to a destination; the length (number of hops) of this path is inversely proportional to $r$. So overall, the contention along this path is now proportional to $r^{2} r^{-1}=r$. This suggests that a low transmission range yields low overall contention along a path, and therefore higher spatial reuse and higher network throughput.

Spatial backoff may also reduce energy consumption. Imagine a single (unicast) transmission; suppose we have one wireless node as transmitter, and several others within its transmission range. Potentially all of the nodes in range can receive the transmission. One transmission occurs at the transmitter and one reception occurs at each of its neighbours, with no discrimination as to whether it is the desired destination. Thus the total energy consumed in this single transmission is $P_{t x}+k P_{r x}$, where $P_{t x}\left[P_{r x}\right]$ is the energy consumed for one transmission [reception], and $k$ is the number of neighbours within transmission range of the transmitter. In spatial backoff where the transmission range is reduced, the number of neighbours that receive the transmission is also reduced, i.e., $k$ is smaller. Therefore, the total energy consumed in the whole system is reduced.

One may conclude that a small transmission range is always desired in a wireless network, but this is not true. The discussion is based on the assumption that the nodes in the network are uniformly distributed and the traffic flows are also uniformly distributed among all the nodes; this may not be the case. A smaller transmission range may not allow the desired destination to be reached in one hop. It may therefore increase the number of hops between a source and destination pair, thus increasing the number of transmissions made by intermediate nodes and associated packet queuing. Furthermore, a problem for all protocols that use power control is how to cope with asymmetric links. Consider Fig. 1 with two nodes $A$ and $B$ where dotted and solid circles represent the range of their high

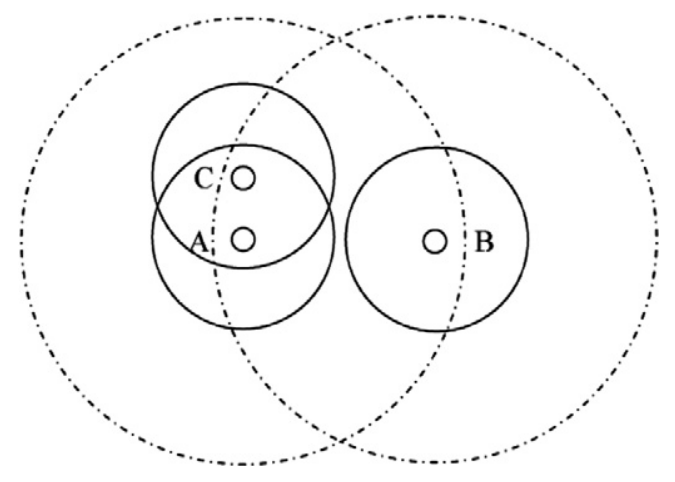

Fig. 1. $B$ may be hidden to $A$ when asymmetric power used.

and low transmission powers, respectively. If $A$ transmits at low power while $B$ transmits at high power, $A$ is within the transmission range of $B$, but the converse is not true. If a handshake is initiated from $A$ to $C$ at low transmission power, $B$ may be a hidden terminal to $A$. The handshake that solves the hidden terminal problem when a common transmission power level is used by all nodes is insufficient in systems where nodes use variablerange transmissions. Hence, whether a low transmit power is desirable may depend on several factors.

We call our approach to backing off in space power backoff ( $\mathrm{PB}$ ) and incorporate it into a CSMA protocol as CSMA/PB. A CSMA/CA protocol, such as IEEE 802.11, may spend a significant amount of time to deliver an individual packet. The contention window starts at a size of 32, and through the binary exponential backoff algorithm may reach a maximum size of 1024 . Indeed, if the contention window is at its maximum size and the packet is still not delivered, the protocol retries a constant number of times at this size before finally giving up on the packet. In comparison, CSMA/ PB backs off through a fixed number of transmission power levels, sensing the carrier at each level. This can be achieved relatively quickly in time, in fact, so much so that it is premature to give up on the packet if the channel is sensed busy at the minimum transmission range; hence, a retry strategy is required. While power backoff alone is effective, even a naïve combination of spatial backoff with temporal backoff proves to be better than CSMA/ CA. We propose three ways of combining backoff in space with backoff in time. We compare the performance of each variant with IEEE 802.11 under the same conditions for a variety of static and mobile ad hoc network scenarios. In all scenarios, 
the throughput per unit energy of CSMA/PB exceeds that of IEEE 802.11, often quite substantially.

The rest of this paper is organized as follows. We summarize related work in Section 2. The basic CSMA/PB protocol is introduced and evaluated in Section 3. Section 4 describes three variants of combined spatial and temporal backoff, assessing the effectiveness of each variant in Section 5. Section 6 analyzes the probability of successful transmission of temporal and spatial backoff strategies. Finally, we present conclusions and directions for future work in Section 7.

\section{Related work}

The analysis of temporal backoff algorithms has been the subject of intense research. There is no simple answer to the question of which is the best algorithm. For example, despite its wide usage in IEEE 802.11 , it is well known that binary exponential backoff results in a provably unstable protocol (infinitely growing queues) under certain modelling assumptions [3]. The existence of stable protocols in this setting also depends on the type of feedback available from the channel and on how the user population is modelled. For acknowledgment-based protocols, Kelly [4] showed that a large class of backoff algorithms, including polynomial backoff, is unstable in the infinite user population model. In contrast, for a finite user population, any super-linear polynomial backoff algorithm has been proved stable, while binary exponential backoff is unstable above a certain arrival rate [5].

Several approaches to medium access control add the dimension of space to transmission. One idea is to use multiple channels with the same aggregate channel capacity thereby spacing out transmissions over channels and not just over time. Nasipuri et al. [6] propose a multichannel MAC protocol using "soft reservation" of the channels. Each node keeps its own channel usage history and preferentially selects a channel used in the past to reduce the contention and collision probability for the same channel among neighbours. In [7] the protocol is extended to select the best channel according to the signal-power on multiple channels so as to distribute the interference on multiple channels evenly. So and Vaidya [8] propose a multichannel MAC that enables nodes to dynamically negotiate channels in order for multiple communications to take place in the same region. Each node maintains a pre- ferred channel list (PCL) that indicates the preference of each channel. The protocol requires a window preceding data packet exchange, during which nodes that want to transmit exchange their PCL information on a common channel and agree on the channel selected by one round of handshake. Multichannel protocols, while requiring that the transceiver is tunable, and the carrier can be sensed on all channels, have improved the overall throughput of CSMA.

Another idea using space for transmission is to use a form of frequency hopping over multiple channels. In hop reservation multiple access (HRMA), Tang and Garcia-Luna-Aceves [9] use a handshake to perform channel reservation for slow frequency hopping spread spectrum networks. All nodes listen using a common frequency hopping sequence. To send data, nodes exchange control packets on the current frequency. If the handshake is successful, they use the same frequency for communication. Other nodes continue hopping and the communicating pair synchronizes with the sequence after the transmission has completed. Tzamaloukas et al. [10] use a similar approach except that the receiver initiates the collision avoidance instead of the sender. Protocols using frequency hopping cannot be applied to systems using forms of direct sequence spread spectrum, e.g., code division multiple access (CDMA) systems, where a signal is spread over a wide bandwidth.

Transmission power control may be applied at the MAC layer to decrease power consumption. Karn [11] incorporated a new field in the IEEE 802.11 handshake to allow a transmitter to specify its transmission power level in the request-to-send (RTS) packet, and the receiver to set the desired transmission power level in the clear-to-send (CTS) packet. The receiver determines the transmission power level based on the required signal-to-noise ratio. The data and acknowledgment (ACK) packets are then transmitted at the power level indicated in the CTS packet. Jung et al. [12] analyze the performance of this scheme and improve it by periodically increasing the transmission power level of the data packet to the maximum power to ensure proper reception of ACK packet. These schemes reduce power consumption at the price of throughput since the RTS/CTS exchange occurs at the highest transmission power level; therefore, the concurrency achieved is at best the same as IEEE 802.11.

Transmission power control may also be applied to increase spectrum reuse. Increasing concurrent 
transmissions around the receiver is the goal of [13-16]; most protocols use an additional control channel. Monks et al. [13] propose the power control multiple access (PCMA) protocol, where an RTS/ CTS-like handshake is used to establish a connection between the transmitter and receiver, but not for silencing neighbours. Instead, a control channel is used for carrier sensing. The receiver periodically sends a busy pulse on the control channel. A potential transmitter listens to the busy tone to determine an upper bound on its transmission power so as to not add too much noise to the existing reception. $\mathrm{Wu}$ et al. [16] combine busy tones and power control. They also use a separate control and data channel together with two busy tone channels, $B T_{t}$ and $B T_{r}$, for transmission and reception, respectively. The receiver sends a busy tone $B T_{r}$ at maximum power. A neighbour can then estimate the channel gain based on the strength of the busy tone and determine whether it is allowed to transmit if its transmission would not add more than a fixed amount of noise; this is similar to [13]. In addition, the transmitter saves energy by sending the data packets and busy tone $B T_{t}$ at reduced power based on the power level of the received CTS. However, neither [13] nor [16] consider additional noise added by future transmitters to the ongoing reception. Muqattash and Krunz [14] propose the power controlled dual channel (PCDC) protocol in which the RTS/CTS packets are transmitted on the control channel, each extended by an additional byte. Using the new byte, the RTS packet indicates the power level used. The receiver determines the channel gain based on the RTS packet it receives and computes a required power level for the transmitter, allowing for a number of future interfering transmissions to take place in its neighbourhood, and puts it in the new byte of CTS packet.

In [15], Muqattash et al. devise the power controlled MAC (POWMAC) protocol to effectively utilize power control on a single channel. However, several aspects of the protocol change with respect to the earlier work in [14]. An access window precedes data transmission, during which other neighbouring nodes within the interference range can exchange RTS/CTS if the interference introduced by the new transmission to the on-going communication is below a fixed signal-to-noise ratio; this provides the possibility of concurrent transmission. Although the use of power control at the MAC layer can increase the channel throughput, most of the protocols need additional hardware, incur overhead, and impose restrictions.

Strongly related to the use of power control to increase spectrum reuse is the use of power control to prevent collisions. Fuemmeler et al. [17] argue that, for CSMA protocols, the product of the transmission power and carrier sense threshold should remain constant. By incorporating this collision prevention condition into a protocol, spatial reuse is improved. In Chu [18], the contention window of IEEE 802.11 is a function of the distance from the transmitter to its next hop destination. A smaller window is used for nodes closer together since the contention in the transmission range required to reach the destination is likely to be reduced. Recently, Yang et al. [19] examined the possibility of increasing system performance by reducing the carrier sense range, while taking into account the MAC overhead. The performance improvement results from the higher level of spatial reuse than is possible with a reduced carrier sense range. In their conclusions Yang et al. [19] suggest as future work adjusting contention based on node access behaviour, even using the term "spatial backoff".

Power control is also utilized by network layer protocols. Among the first routing protocols to use power control is power-aware routing (PARO) [20]. It minimizes the transmission power needed to forward packets between wireless nodes in ad hoc networks. In the common power (COMPOW) protocol [21] all the nodes converge to a universal power level selected to be the minimum power level that keeps the network connected and the power consumption minimal. However, if the nodes in the network are not uniformly distributed significant energy is wasted. The clustered power (CLUSTERPOW) protocol [22] handles this problem by running several routing agents, each corresponding to a different power level, in every node. Every node forwards a packet to a destination using the smallest power level to reach the destination. The drawback for this protocol is the overhead to maintain all the routing tables.

Further, there are well-known interactions among protocols (see [23] and references therein). Indeed with power control these interactions are likely to be more tightly coupled. Interesting questions relate to which protocol should be responsible for selecting the transmission power level. Techniques such as those suggested in Vadde et al. [24] to optimize interactions may apply, but these questions are out-of-scope of this paper. 


\section{Carrier sense multiple access with power backoff (CSMA/PB)}

In this section we introduce the basic form of our carrier sense multiple access with power backoff (CSMA/PB) protocol, and provide a simulationbased evaluation of throughput and energy consumption. We begin by describing fundamental assumptions about the network and CSMA protocols.

\subsection{Fundamental assumptions}

Throughout the remainder of this paper, we assume that each node of the network is identically equipped with an omni-directional antenna and a half-duplex transceiver operating on a single channel; thus, when a packet is transmitted, it is received by all (non-transmitting) nodes within the transmission range of the transmitter. We further assume that the radio transceiver in each node can be tuned to a number of discrete power levels, with each power level naturally corresponding to a unique transmission range. The minimum and maximum power levels are denoted by $P_{\min }$ and $P_{\max }$ respectively. In addition, we assume that the tuning of a transceiver to a particular transmit power level can be accomplished on a per-packet basis and that tuning to a particular power level does not involve any significant cost.

Most CSMA protocols assume that the links are bidirectional (i.e., symmetric). That is, communication is possible between nodes $i$ and $j$ if and only if the transmission power levels of $i$ and $j$ are high enough that $j$ can receive from $i$, and $i$ can receive from $j$. If nodes are all transmitting at the same power level, then this is a natural assumption, though even then unidirectional links may exist due to noise, interference, etc. When using power control (and in contrast to the situation with typical existing CSMA protocols) nodes often transmit at differing power levels, and unidirectional links may occur. This asymmetry is addressed in the CSMA/ PB protocol design by adjusting transmission powers as necessary to avoid unidirectional links whenever it is reasonable to do so.

\subsection{The basic CSMAIPB protocol}

Using CSMA/PB, the transmission of each data packet follows a four-way handshake with some added elements related to power control. To describe the protocol, we consider a node $s$ that transmits a series of data packets. Let $p_{i}$ be the power level utilized by $s$ in transmitting the $i$ th data packet.

Suppose there are $n$ transmission power levels, $P_{\max }=n$ and $P_{\min }=1$. Initially, the transmission power level of $s$ is set to the maximum power level (i.e., $\left.p_{1} \leftarrow P_{\max }\right)$. To transmit the $i$ th data packet, $s$ first senses the channel; if the channel is busy, the node updates the network allocation vector (NAV) as in IEEE 802.11. If the channel is free, $s$ transmits an RTS at power level $p_{i}$. That RTS includes the power level $p_{i}$ being used to transmit the packet. Following that transmission there are two cases:

(i) If $s$ subsequently receives the corresponding CTS, then the $i$ th data packet is transmitted at power level $p_{i}$. If the transmission is successful, then $s$ receives an ACK.

(ii) If $s$ does not receive a CTS, then the RTS may have been involved in a collision. In this case, the current transmission power level $\left(p_{i}\right)$ of $s$ is reduced and $s$ sends a new RTS at a reduced power level if the channel is free.

The difference between our power backoff and temporal backoff is that when a backoff is needed, temporal backoff increases the contention window size and waits for a period related to the window size. Power backoff reduces the transmission power level. Both actions reduce contention, the first in the temporal domain, the second in the spatial domain.

The complete basic CSMA/PB transmitter and receiver protocols appear in Figs. 2 and 3, respectively.

Similar to Karn [11], the power level $p_{i}$ is included in the RTS. This is to avoid problems arising from unidirectional links that may result when the transmitter and receiver use different transmission power levels. By embedding the power level $p_{i}$ in the RTS sent by $s$, when node $r$ receives that RTS, it can set the transmission power level for sending the CTS packet to the level embedded in the RTS packet that it received. Likewise, $r$ will use that same power level for the ACK packet that ends a successful transmission.

An important issue in CSMA/PB is determining the appropriate transmission power level for the next packet (packet $i+1$ ) in the series after a successful packet exchange. In IEEE 802.11, the contention window is reset to its minimum size after 


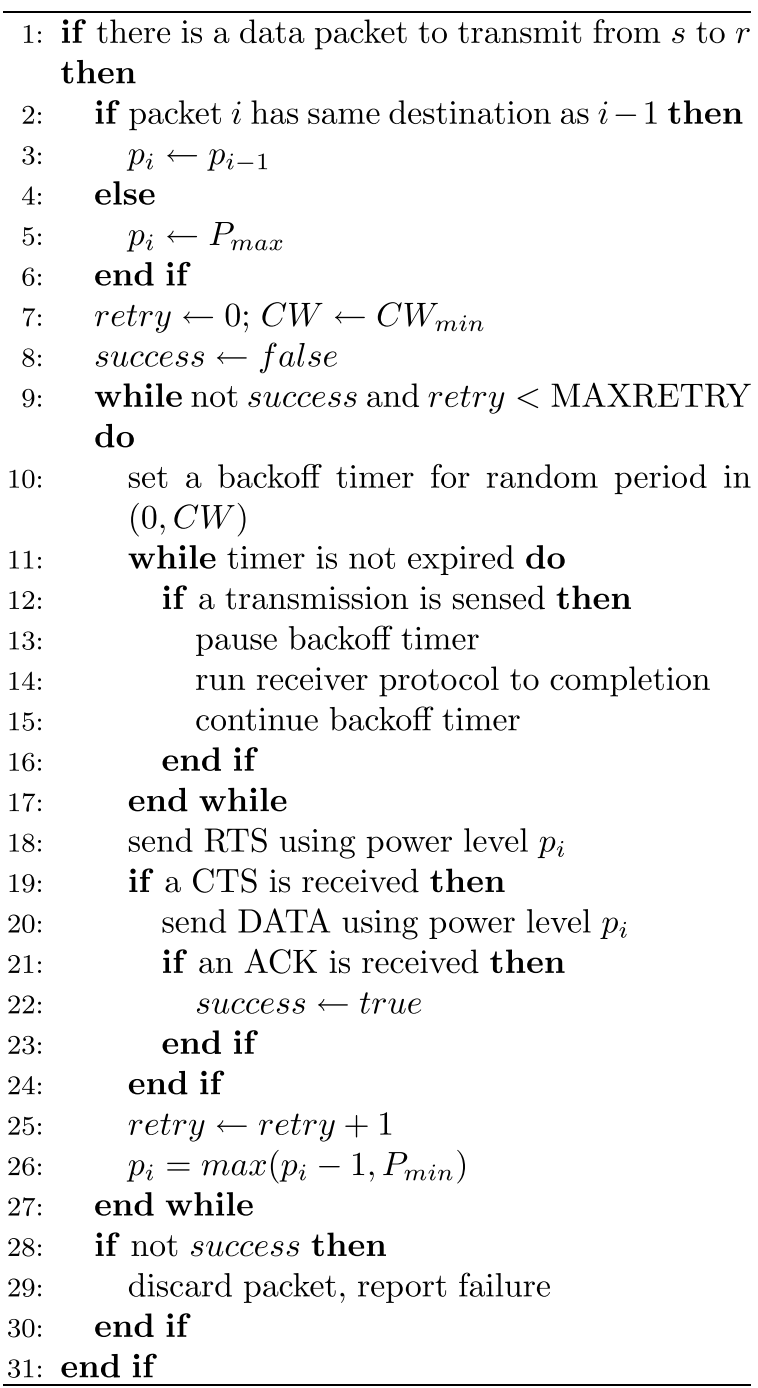

Fig. 2. Basic CSMA/PB transmitter protocol.

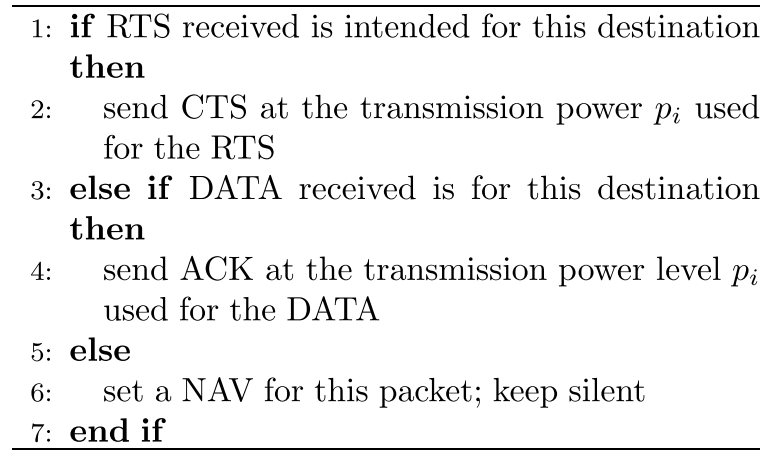

Fig. 3. Basic CSMA/PB receiver protocol.

each successful four-way handshake. In CSMA/PB, the value of $p_{i+1}$ depends on the next hop destination of packet $i+1$. If that destination is the same as that of packet $i$, then the transmission power level $p_{i}$ is retained as the value of $p_{i+1}$. If the destination is different, then the value of $p_{i+1}$ is initialized to $P_{\max }$.

As in IEEE 802.11, when a neighbour $z$ of $s$ or $r$ overhears an RTS or a CTS packet associated with the transmissions of $s$ or of $r$, then node $z$ sets its NAV for the duration of the data-ACK transmission.

One potential difficulty with the CSMA/PB protocol is that a node may be "unlucky" and always sense a busy channel. In this case, the node repeatedly decrements its transmission power level, eventually setting its transmission power to the minimum possible level. A retry strategy is followed, with the node able to reach a MAXRETRY number of attempted transmissions before the packet is dropped. In the basic CSMA/PB, the contention window $(\mathrm{CW})$ is fixed at 32 (the minimum in IEEE 802.11) and is never changed in the protocol. The only purpose of this contention window is to introduce a small amount of jitter in the retry in case nodes become synchronized.

\subsection{Evaluation of the basic CSMA/PB}

We evaluate the basic CSMA/PB protocol using $n=3$ transmission power levels, comparing its performance to IEEE 802.11, in the $\mathrm{ns}-2$ network simulator version 2.26 [25]. Table 1 shows some important simulation parameters. The carrier sense threshold and receive threshold are set to the default values in $n s-2$ and are constant. There are existing power control schemes that use carrier sense threshold tuning algorithms (e.g. [19]) or even disable the physical carrier sensing (e.g. [26]), however according to recent work by Kim et al. [27] tuning transmission power yields higher network capacity than

Table 1

Simulation parameters

\begin{tabular}{ll}
\hline Data packet size & 1000 bytes \\
Transport protocol & UDP \\
Traffic arrival rate & $0.5 \mathrm{Mbps}$ \\
Channel data rate & $1 \mathrm{Mbps}$ \\
Simulation time & $200 \mathrm{~s}$ \\
Antenna & Omni-directional \\
Carrier sense threshold & $1.559 \mathrm{E}-11 \mathrm{~W}$ \\
Receive threshold & $3.652 \mathrm{E}-10 \mathrm{~W}$ \\
Transmission power level 3 & $0.2818 \mathrm{~W}(250 \mathrm{~m})$ \\
Transmission power level 2 & $7.214 \mathrm{E}-3 \mathrm{~W}(100 \mathrm{~m})$ \\
Transmission power level 1 & $8.5872 \mathrm{E}-4 \mathrm{~W}(40 \mathrm{~m})$ \\
\hline
\end{tabular}


tuning carrier sense threshold. In this paper, we vary the transmission power variable and hold the carrier sensing threshold constant. Since the packet reception model in $n s-2$ is simplistic [28], the simulation results of $n s-2$ can be optimistic. Multiple runs of simulations with randomly generated seeds have been performed to ensure a $95 \%$ confidence for all the data points with intervals of less than $5 \%$ that plotted.

The network scenario consists of a $250 \times 500 \mathrm{~m}$ rectangle with 50 nodes, each moving according to the (steady-state initialized) random way-point mobility model at $2 \mathrm{~m} / \mathrm{s}$ with a $2 \mathrm{~s}$ pause time [29]. We randomly select four nodes to generate two flows for a low traffic case, and 10 nodes to generate five flows for a high traffic case.

We compare the protocols using two metrics: the total throughput (the total amount of data delivered by all the flows in the network) and the throughput per unit energy (the total throughput divided by the total amount of energy spent by all the nodes in the network).

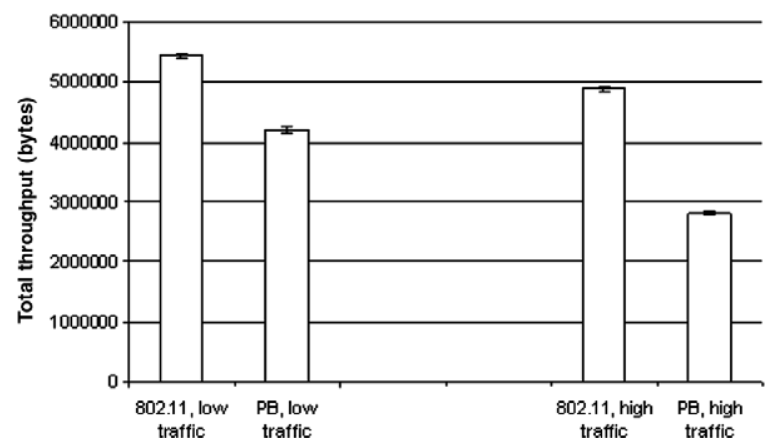

Fig. 4. Total throughput for IEEE 802.11 and basic CSMA/PB for a low and a high traffic scenario.

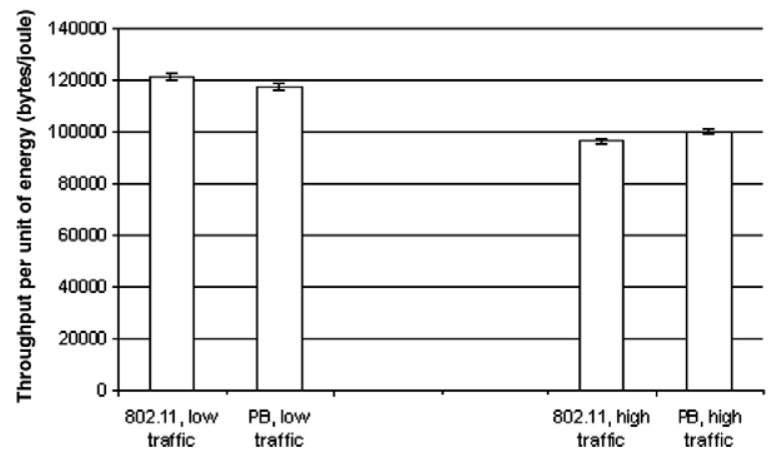

Fig. 5. Throughput per unit of energy for IEEE 802.11 and basic CSMA/PB for a low and a high traffic scenario.
Fig. 4 shows disappointing total throughput for basic CSMA/PB compared to IEEE 802.11 in both the low and the high traffic scenarios. More promising however, are the results in Fig. 5, showing comparable throughput per unit of energy of the two protocols. While IEEE 802.11 may spend a significant amount of time to deliver an individual packet, CSMA/PB backs off in space relatively quickly in time and may give up on a packet prematurely. These observations suggest that the throughput of CSMA/PB may improve if we revisit the element of time in our backoff strategy.

\section{Extending CSMA/PB: combining spatial and temporal backoff}

While spatial backoff alone is promising, better adaptation in some scenarios is required. For example, in a dense network with a large number of active transmitters, most of the transmitters back off to the minimum transmission power level. There is no action that can be taken by basic CSMA/PB to alleviate continued contention. This scenario motivates combining spatial backoff with temporal backoff.

\subsection{Spatial followed by temporal backoff}

One way to combine spatial backoff with temporal backoff is to follow one approach by the other. Fig. 6 shows a transmitter with $n=3$ transmission power levels, first backing off in space. Once the minimum power level is reached, the transmitter then backs off in time using binary exponential backoff. The size of the contention window doubles, permitting nodes to use the time dimension once the space dimension is exhausted. Retransmissions always occur at the minimum power level. Spatial followed by temporal backoff is accomplished by replacing line 26 in Fig. 2 with the statements in Fig. 7. The contention window is reset (line 7 in

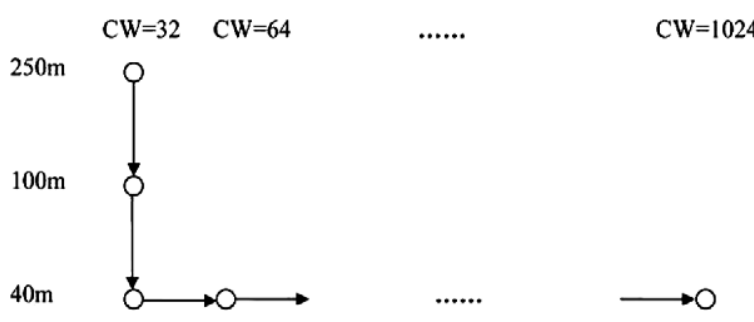

Fig. 6. Spatial followed by temporal backoff. 


$$
\begin{aligned}
& \text { 1: if } p_{i} \neq P_{\min } \text { then } \\
& \text { 2: } \quad p_{i} \leftarrow p_{i}-1 \\
& \text { 3: else } \\
& \text { 4: } \quad C W \leftarrow \min \left(C W \times 2, C W_{\text {max }}\right) \\
& \text { 5: end if }
\end{aligned}
$$

Fig. 7. To implement spatial followed by temporal backoff replace line 26 in Fig. 2 by these statements.

Fig. 2) to the minimum size whenever a new packet arrives.

\subsection{Alternating spatial and temporal backoff}

Another way to combine spatial backoff with temporal backoff is to alternate approaches. Fig. 8 illustrates backing off in space followed by backing off in time. When the minimum transmission power level is reached, the size of the contention window is doubled. Spatial backoff is then run using the larger contention window. This alternating sequence may be repeated until the contention window reaches its maximum size. Spatial backoff alternating with temporal backoff is accomplished by replacing line 26 in Fig. 2 with the statements in Fig. 9.

In this combined approach asymmetric links are more likely to arise. This is because each step of backoff can involve a change in the transmission power level. A copy mechanism may be used to alleviate this problem. In this variant of the protocol, whenever a node overhears a transmission, it sets its transmission power level to the minimum of the
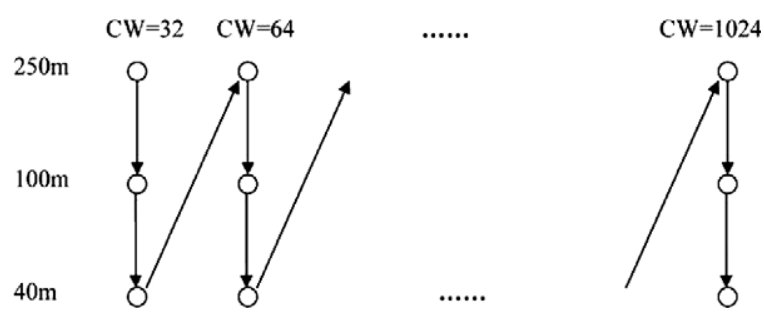

Fig. 8. Alternating spatial and temporal backoff.

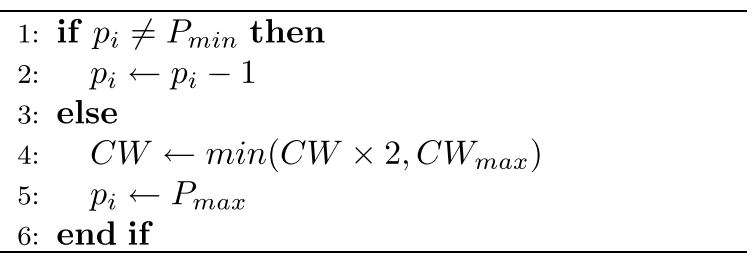

Fig. 9. To implement alternating spatial and temporal backoff replace line 26 in Fig. 2 by these statements. overheard packet and its current transmission power level.

\subsection{Alternating temporal and spatial backoff}

Of course, backoff could instead occur in the temporal domain first. This is illustrated in Fig. 10. In order for such an approach to be practical, the maximum contention window size $C W_{\max }$ is reduced to 256 from 1024 used in IEEE 802.11. As before, replacing line 26 in Fig. 2 by the statements in Fig. 11 implements the alternation of temporal and spatial backoff.

\subsection{Discussion}

Of these three approaches to combining spatial and temporal backoff, in an active and dense network, the first approach aggressively reduces the transmission power to the minimum level. If sources and destinations of flows are far apart, this encourages longer paths with short hops to be utilized.

When alternating approaches to backoff, asymmetric links are more likely to arise when backing off in the spatial domain first. Nodes using high transmission power levels have an advantage in gaining access to the channel over nodes using low transmission power levels because high power nodes may be hidden to low power nodes (recall Fig. 1). Starting the alternation by backing off in time is the most conservative combination, essentially

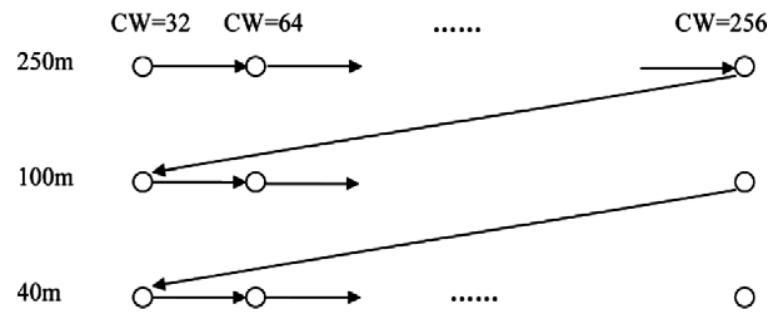

Fig. 10. Alternating temporal and spatial backoff.

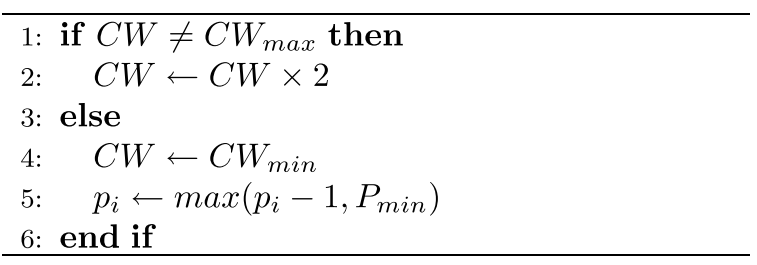

Fig. 11. To implement alternating temporal and spatial backoff replace line 26 in Fig. 2 by these statements. 
running IEEE 802.11 at each of the $n$ transmission power levels.

\section{Evaluation of the extensions of CSMA/PB}

We evaluate the three extensions of CSMA/PB presented in Section 4, comparing their performance to IEEE 802.11 in the $\mathrm{ns}-2$ network simulator [25]. The simulation parameters used are the same as used in the evaluation of basic CSMA/PB (see Table 1); $n=3$ transmission power levels are assumed. The variants of CSMA/PB use: spatial followed by temporal backoff ("direct"); alternating spatial and temporal backoff ("power first"); alternating temporal and spatial backoff ("time first"); and "power first" with a copy mechanism (PFwC) to alleviate asymmetric links.

\subsection{Power-aware routing}

If the routing protocol does not take into account variation in transmission power levels it will use unnecessary paths when the transmission power is high and have no path to use when the transmission power is low. Therefore, routing must be poweraware.

Let $w\left(v_{i}, v_{j}\right)$ denote the transmission power needed for node $v_{i}$ to transmit in one hop to $v_{j}$; this is infinity if no such power level exists. Then for each $s-t$ flow and each transmission power level $\rho$, $1 \leqslant \rho \leqslant n$, a path $s=v_{0}, v_{1} \ldots v_{k}=t$ is found such that $w\left(v_{0}, v_{1}\right) \leqslant \rho$ and $\rho+\sum_{j=1}^{k-1} w\left(v_{j}, v_{j+1}\right)$ is minimized. Fig. 12 illustrates the idea of the poweraware routing protocol.

A two-dimensional routing table $T[1 \ldots(N-$ $1), 1 \ldots n]$ at each node contains entries for each of the other $N-1$ destinations at each of the $n$ power levels. $T[t, \rho]$ specifies the next-hop node on the path

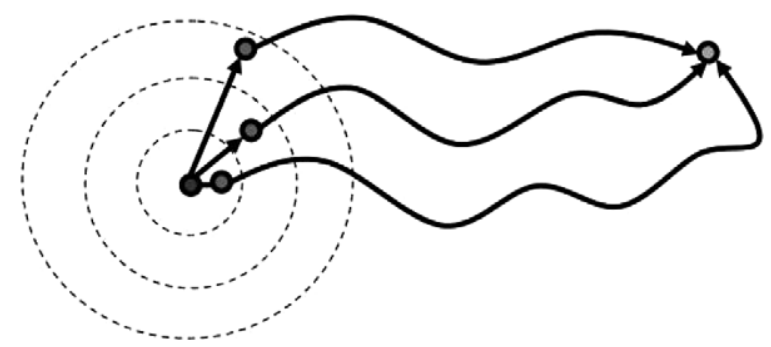

Fig. 12. Idea of power-aware routing: The cost of the first hop depends on the transmission power level. Total power consumption is then minimized. to $t$ at transmission power level $\rho$; if the path weight is infinity then there is no path whose first hop is at power $\rho$. If $n=1$ (as in IEEE 802.11), then the computed path is a minimum hop-count path.

In this power-aware routing protocol, if node $v_{i}$ receives a packet to forward towards destination $t$, the next hop node $v_{i+1}$ depends on the transmission power level $\rho$ used by $v_{i}$. Node $v_{i}$ selects $v_{i+1}=T[t, \rho]$ as the next hop node. The value of $\rho$ is determined by CSMA/PB. If CSMA/PB changes the transmission power level then this may change the next hop node through which the packet is routed.

We illustrate the operation of the routing protocol using the sample network in Fig. 13. Assume that there are $n=3$ transmission power levels. The cost of a link using transmission power level $i$ is equal to $i$ for $i=1, \ldots, 3$. For simplicity, assume that there are no other links except those shown in the figure.

Suppose that we want to route a packet from node $A$ to node $E$. For the intermediate transmission power level $(\rho=2)$, the candidate next-hop nodes with first-hop less than or equal to transmission power level 2 are nodes $B$ and $C$. Via node $B$, the minimum weight path is $A B E$ with $w(A, B)+w(B, E)=2+3=5$. Via node $C$, the minimum weight path is $A C E$ with $w(A, C)+w(C, E)=$ $2+3=5$. Therefore the next-hop node for destination $E$ using transmission power level 2 is $C$ ( $B$ is equally good in this small example). A route from node $A$ to node $E$ using the highest transmission power level has candidate next-hop nodes of $B, C$, and $D$, among which the minimum cost path is $A D E$ with $w(A, D)+w(D, E)=3+2=5$. Thus, the entry in the routing table for node $A$ is $T[E, 3]=D$. Table 2 shows the complete routing table for node $A$.

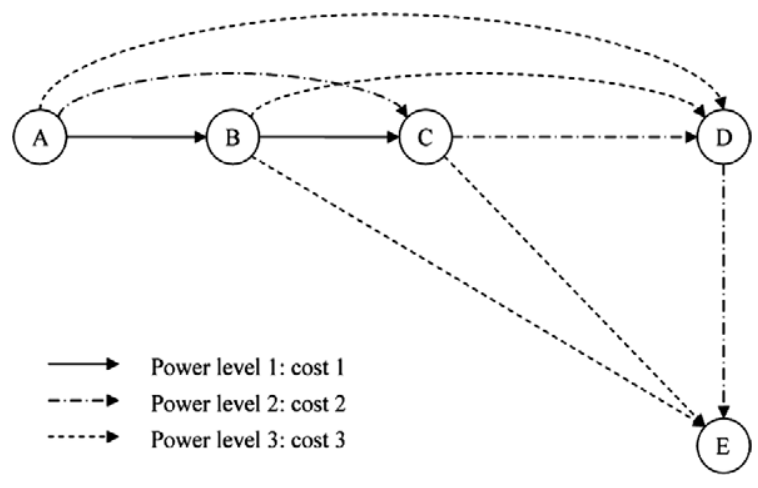

Fig. 13. A sample network to illustrate the idealized power-aware routing protocol. 
Table 2

Routing table for node $A$ for the sample network of Fig. 13

\begin{tabular}{llll}
\hline Destination & Next hop at & Next hop at & Next hop at \\
node & $\rho=1$ & $\rho=2$ & $\rho=3$ \\
\hline B & B & B & B \\
C & B & C & C \\
D & B & C & D \\
E & B & C & D \\
\hline
\end{tabular}

It is possible that for some source $s$ and destination $t$, there is no next-hop node for a given transmission power level $\rho$. In this case, the entry $T[t, \rho]$ in the routing table at $s$ is set to infinity. In our implementation, CSMA/PB skips these transmission power levels as no next-hop node is available.

Our implementation of the power-aware routing protocol is centralized. It was designed to explore the potential of a MAC protocol that utilizes transmission power control. The design of a distributed power-aware routing protocol for this purpose is out-of-scope of this paper.

In the following several subsections we compare the performance of CSMA/PB and IEEE 802.11 under increasingly complex network topologies.

\subsection{Static chain topology}

We first consider two scenarios for a static chain topology where ten nodes are arranged in a line $30 \mathrm{~m}$ apart (see Fig. 14). The first scenario has three single-hop flows from nodes 1,5 , and 9 to nodes 2 ,

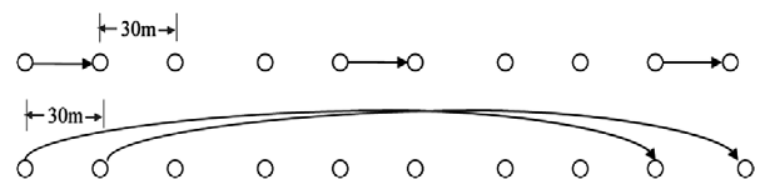

Fig. 14. Chain topology with single-hop and multi-hop flows.

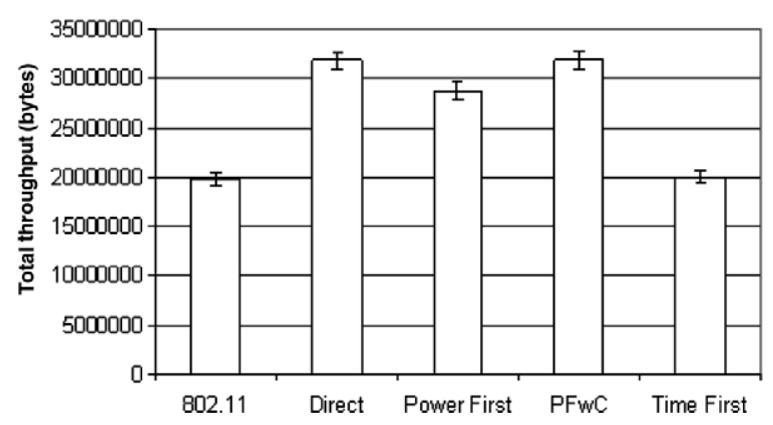

Fig. 15. Throughput in chain topology with single-hop flows.
6 , and 10, respectively. The second scenario has two multi-hop flows from nodes 1 and 2 to nodes 9 and 10 , respectively.

Fig. 15 plots throughput in the single-hop flow scenario. All variants of CSMA/PB outperform IEEE 802.11 except "time first" which decreases transmission power too slowly to exploit the potential for spatial reuse. In this scenario, all three flows can transmit simultaneously if the lowest transmission power is used. The "power first with copy" performs the best, as the nodes reach the lowest transmission power level quickly. The copy mechanism accelerates this decrease since once a node successfully transmits at minimum power, the overhearing neighbours copy this power level. The "direct" protocol does not use the copy mechanism and therefore takes slightly longer for all the nodes to reach the minimum transmission power.

Fig. 16 shows larger absolute differences in throughput per unit energy. Here, significant gains can be seen; even "time first" is $30 \%$ higher than IEEE 802.11. Table 3 shows the number of packets transmitted at each power level in each of the five protocols. The packets transmitted at lower transmission power levels yield significant gains in energy and throughput per unit energy.

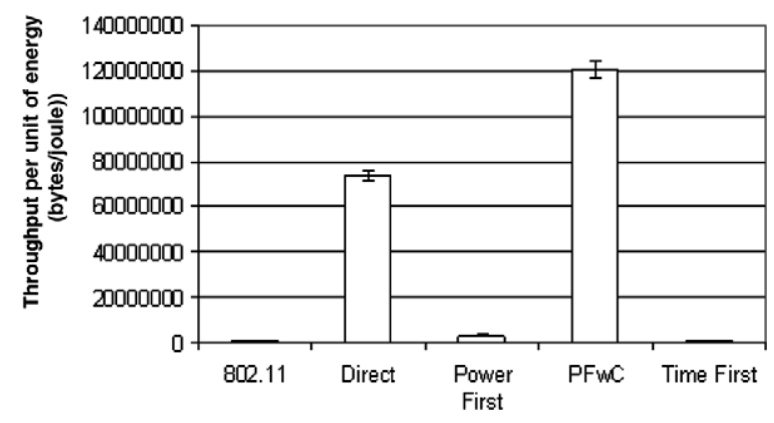

Fig. 16. Throughput/energy in chain topology with single-hop flows.

Table 3

Number of packets transmitted at each transmission power level, chain topology, single-hop flows

\begin{tabular}{llll}
\hline Protocol & $\begin{array}{l}0.2818 \mathrm{~W} \\
(250 \mathrm{~m})\end{array}$ & $\begin{array}{l}7.214 \mathrm{E}-3 \mathrm{~W} \\
(100 \mathrm{~m})\end{array}$ & $\begin{array}{l}8.5872 \mathrm{E}-4 \mathrm{~W} \\
(40 \mathrm{~m})\end{array}$ \\
\hline IEEE 802.11 & 81,102 & 0 & 0 \\
Direct & 8 & 11,388 & 113,381 \\
Power first & 12,274 & 55,139 & 56,604 \\
Power first & 12 & 78 & 124,876 \\
$\quad$ with copy & & & 5051 \\
Time first & 65,838 & 18,179 & \\
\hline
\end{tabular}




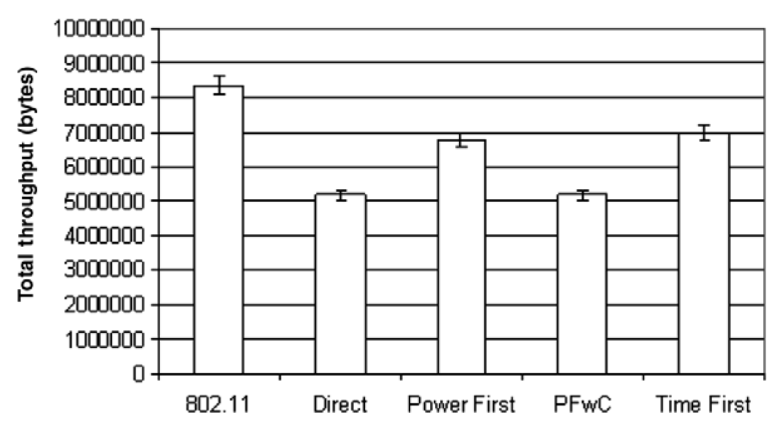

Fig. 17. Throughput in chain topology with multi-hop flows.

Fig. 17 shows the throughput achieved in the chain topology with multi-hop flows. All variants of CSMA/PB suffer because the multi-hop flows compete with each other. Nodes using higher transmission power levels gain access to the channel over nodes using lower transmission power. In "power first" and "time first," nodes can use high transmission power more frequently than the other two CSMA/PB protocols, thus their throughput is higher. However, as expected, they also consume more energy.

Despite this, Fig. 18 shows that all variants of CSMA/PB obtain higher throughput per unit energy than IEEE 802.11. Table 4 shows the number of packets transmitted at each power level. "Direct" and "power first with copy" consume the least energy; while their throughput suffers from taking short hops to the destination they achieve better throughput per unit energy.

\subsection{Static cluster topology}

Next we consider a static cluster topology in which there are two groups of five nodes, each with two intra-group flows between nodes $30 \mathrm{~m}$ apart,

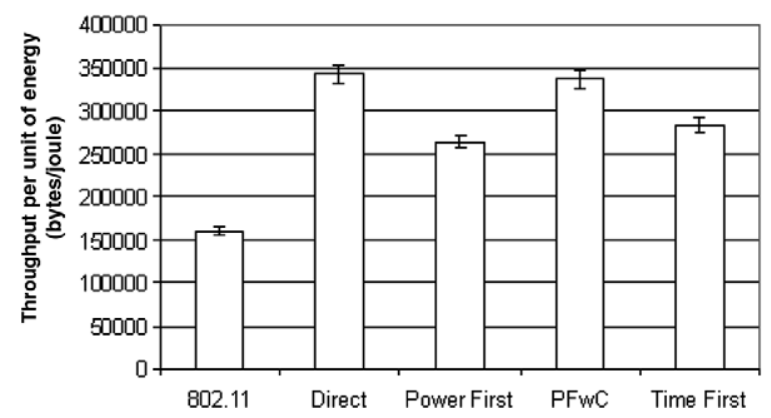

Fig. 18. Throughput/energy in chain topology with multi-hop flows.
Table 4

Number of packets transmitted at each transmission power level, chain topology, multi-hop flows

\begin{tabular}{llll}
\hline Protocol & $\begin{array}{l}0.2818 \mathrm{~W} \\
(250 \mathrm{~m})\end{array}$ & $\begin{array}{l}7.214 \mathrm{E}-3 \mathrm{~W} \\
(100 \mathrm{~m})\end{array}$ & $\begin{array}{l}8.5872 \mathrm{E}-4 \mathrm{~W} \\
(40 \mathrm{~m})\end{array}$ \\
\hline IEEE 802.11 & 79,635 & 0 & 0 \\
Direct & 22,636 & 8186 & 66,445 \\
Power first & 39,425 & 21,237 & 34,363 \\
Power first & 23,535 & 20,204 & 60,051 \\
$\quad$ with copy & & & \\
Time first & 36,908 & 27,033 & 22,023 \\
\hline
\end{tabular}

and a single inter-group flow between a pair of nodes $200 m$ apart (see Fig. 19).

Fig. 20 shows throughput for the static cluster topology. All variants of CSMA/PB except "time first" obtain higher throughput than IEEE 802.11. With spatial backoff, concurrency within clusters leads to higher throughput. Fig. 21 shows that "direct" and "power first with copy" obtain the highest throughput per unit energy. These values are dramatically higher than those for 802.11. Table 5 shows the number of packets transmitted at each transmission power level for this topology.

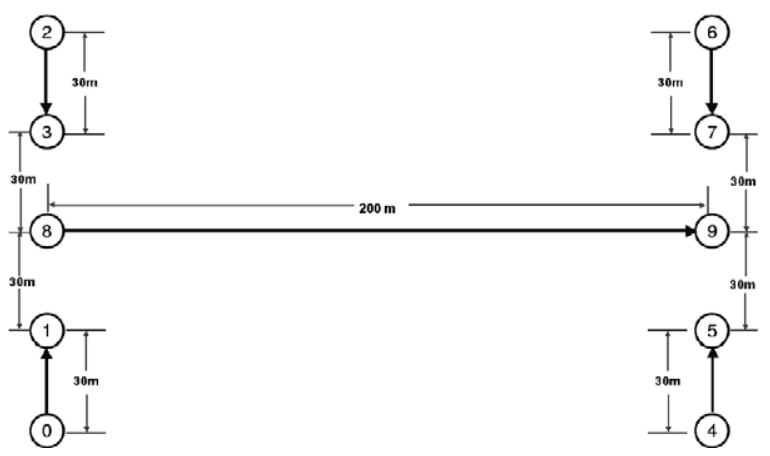

Fig. 19. Static cluster topology.

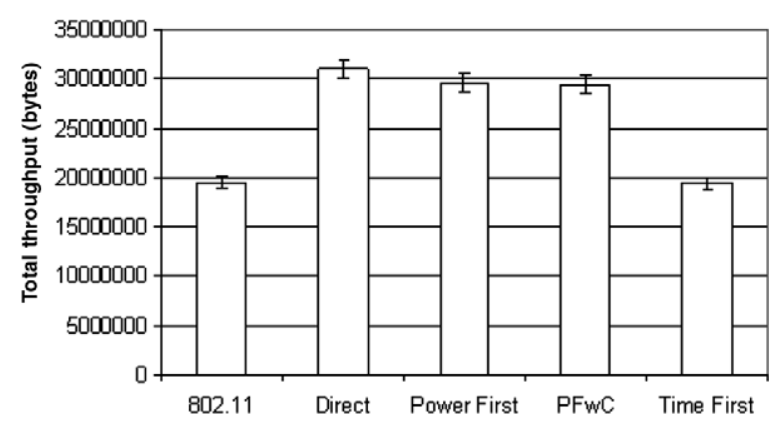

Fig. 20. Throughput in static cluster topology. 


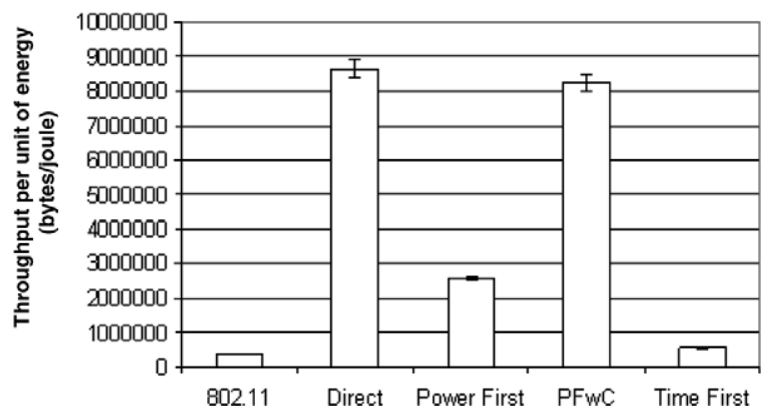

Fig. 21. Throughput/energy in a static cluster topology.

Table 5

Number of packets transmitted at each transmission power level, static cluster topology

\begin{tabular}{llll}
\hline Protocol & $\begin{array}{l}0.2818 \mathrm{~W} \\
(250 \mathrm{~m})\end{array}$ & $\begin{array}{l}7.214 \mathrm{E}-3 \mathrm{~W} \\
(100 \mathrm{~m})\end{array}$ & $\begin{array}{l}8.5872 \mathrm{E}-4 \mathrm{~W} \\
(40 \mathrm{~m})\end{array}$ \\
\hline IEEE 802.11 & 83351 & 0 & 0 \\
Direct & 796 & 57,583 & 83,528 \\
Power first & 11,433 & 99,412 & 16,040 \\
$\begin{array}{l}\text { Power first } \\
\quad \text { with copy }\end{array}$ & 1361 & 102,804 & 29,629 \\
Time first & 22,560 & 84,014 & 15,726 \\
\hline
\end{tabular}

\subsection{Mobile ad hoc network}

In this scenario we consider a mobile ad hoc network (MANET) with 60 nodes in a $500 \times 250 \mathrm{~m}$ area. There are 50 nodes, each moving according to the (steady-state initialized) random way-point mobility model at $2 \mathrm{~m} / \mathrm{s}$ with a $2 \mathrm{~s}$ pause time [29]. Between the remaining ten nodes, we establish five flows, one from each of five fixed sources positioned evenly along the left-hand edge of the rectangular area to destinations positioned directly across on the right-hand side of the area (see Fig. 22).

Fig. 23 shows the throughput in this scenario. All variants of CSMA/PB obtain higher throughput than IEEE 802.11. This is no surprise since in IEEE

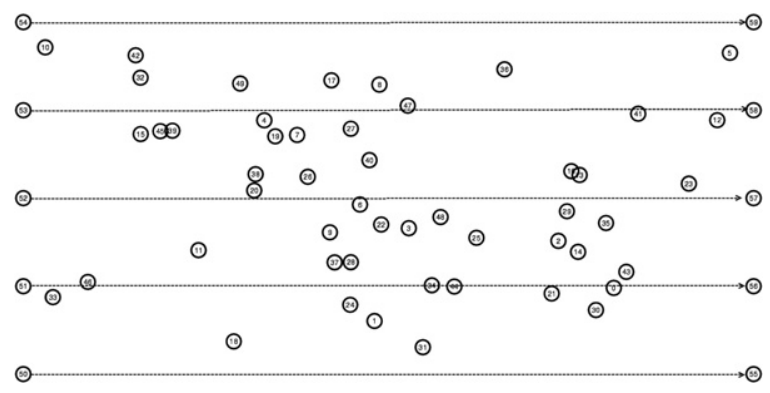

Fig. 22. Multi-hop flows in a mobile ad hoc network.

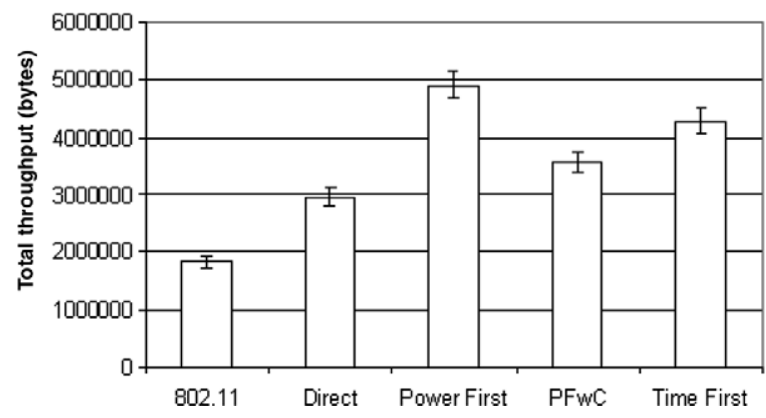

Fig. 23. Throughput in a MANET with multi-hop flows.

802.11 there is no concurrency among the five flows, while in CSMA/PB the flows can run concurrently. Among the CSMA/PB variants, "direct" suffers the most since in this protocol nodes tend to back off to minimum power very often, increasing the number of hops along the path. The same is true of "power first with copy" and "time first," except that they are less aggressive in backing off in power, so each obtains a slight increase in throughput. The throughput for "power first" is unexpectedly high. While this variant of CSMA/PB can suffer from a large number of asymmetric links, this seems to be an advantage when the network is busy and all of the flows are multi-hop. At any given time retries from nodes with high transmission power levels are more likely to succeed because they literally over-power nodes with low transmission power.

Compared to IEEE 802.11, there are fewer nodes transmitting at high power, and thus there are fewer nodes competing for the channel. Furthermore, since in "power first", a node's contention window increases only after a round of power backoff, the average contention window size is expected to increase slower than IEEE 802.11. These observations likely explain why "power first" outperforms IEEE 802.11. The throughput per unit energy of all variants of CSMA/PB dramatically outperform IEEE 802.11 in the mobile scenario (see Fig. 24). Table 6 shows the number of packets transmitted at each power level.

\subsection{Summary}

The simulation results in this section demonstrate convincingly the effectiveness of using the dimension of space in the backoff strategy. The power backoff protocols show excellent throughput per unit energy when compared to IEEE 802.11. The "direct" and "power first with copy" are aggressive protocols. 


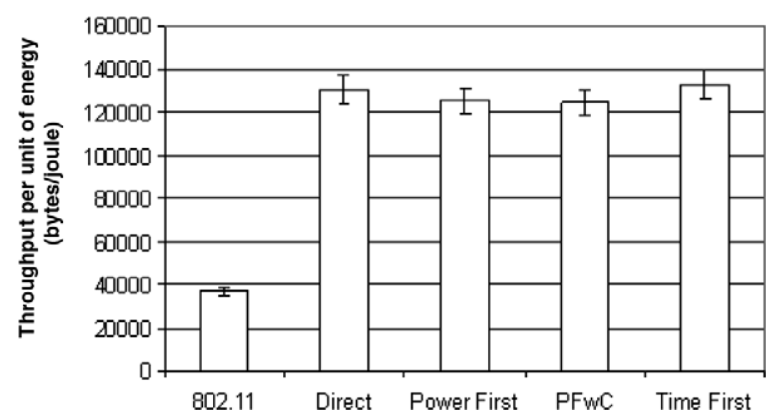

Fig. 24. Throughput/energy in a MANET with multi-hop flows.

Table 6

Number of packets transmitted at each transmission power level, mobile ad hoc scenario

\begin{tabular}{llll}
\hline Protocol & $\begin{array}{l}0.2818 \mathrm{~W} \\
(250 \mathrm{~m})\end{array}$ & $\begin{array}{l}7.214 \mathrm{E}-3 \mathrm{~W} \\
(100 \mathrm{~m})\end{array}$ & $\begin{array}{l}8.5872 \mathrm{E}-4 \mathrm{~W} \\
(40 \mathrm{~m})\end{array}$ \\
& $250 \mathrm{~m}$ & $100 \mathrm{~m}$ & $40 \mathrm{~m}$ \\
\hline IEEE 802.11 & 73,408 & 0 & 0 \\
Direct & 26,716 & 57,135 & 35,977 \\
Power first & 58,704 & 25,842 & 4286 \\
Power first & 55,651 & 26,685 & 6618 \\
$\quad$ with copy & & & \\
Time first & 41,082 & 53,807 & 12,149 \\
\hline
\end{tabular}

In the scenarios examined, they always perform among the best in throughput per unit of energy benefitting from the energy saved by utilizing small transmission power levels. The "power first" variant can suffer from creating asymmetric links, however this seems to be an advantage in some mobile multi-hop scenarios. The "time first" is the most conservative variant of CSMA/PB. Its performance is similar to IEEE 802.11 if the channel is not busy. In a busy and dense multi-hop network, it is among the best strategies to use.

While power backoff protocols are successful on improving throughput and throughput per unit of energy globally, fairness issues arise from using variable power control. From Table 6 we see that in a general mobile ad hoc network scenario, the number of packets transmitted at a high power level are more than that transmitted at a low power level, suggesting that high power transmissions are more likely to be successful than low power transmissions. This can be explained by the new hidden terminal problems that arise from asymmetric links (recall Fig. 1). We analyze this problem in more detail in the next section. In this paper our goal is to optimize throughput and throughput per unit of energy without regard to fairness.

\section{Probability of success: temporal versus spatial backoff strategies}

In this section we analyze the probability of successful transmission using a temporal backoff strategy and also a spatial backoff strategy.

\subsection{Temporal and spatial backoff strategies}

The main goal of backoff is to resolve contention and reduce the probability of collisions. We measure the effectiveness of temporal backoff and spatial backoff by $P_{\text {success }}$, the probability of a node having a successful transmission in the current transmission frame.

Assuming a CSMA-based protocol, a transmission frame consists of a two-way or a four-way handshake: RTS-CTS-data-ACK or data-ACK. For simplicity, we consider a two-way handshake. If the data and the ACK are both received properly, the transmission is successful. We use this probability as the metric because the average $P_{\text {success }}$ in the network represents the throughput for the network. The higher this probability, the higher the network throughput.

We use the following notation:

$P_{\text {transmit. }}$ The probability of transmission; this represents the probability of a node transmitting at the beginning of the current transmission frame.

$P_{\text {idle. }}$ The probability that the channel is idle.

$P_{\text {silent. The probability of a node remaining silent }}$ in the current transmission frame.

$R_{\mathrm{i}}$. The transmission range corresponding to transmission power level $i$; $R_{\max }$ is the maximum transmission range.

$D$. The density of nodes in the network.

$P_{\text {active }}$. The probability of nodes in the network being active, i.e., they have packets (either data or control packets) queued for transmission.

First we compute $P_{\text {success }}$ for a temporal backoff strategy. In the CSMA protocol, in order for a packet to be transmitted correctly from the source node $s$ to the (one-hop) destination node $t$, two conditions must be satisfied:

(i) The source node $s$ transmits in the current transmission frame, i.e., its backoff timer expires before this frame starts. This condition ensures the initiation of the handshake. 
(ii) The channel is idle around the destination node $t$, i.e., none of its neighbours is transmitting. In this case, the data packet is decoded correctly resulting in an ACK packet as a response.

All nodes, except $t$, receiving the data packet sent by $s$ update their NAV and remain silent. This allows $s$ to successfully receive the ACK (for simplicity, we do not consider nodes moving into or out of the transmission range of node $s$ ). If these two conditions are satisfied, a handshake is completed successfully. Therefore,

$P_{\text {success }}=P_{\text {transmit }} \times P_{\text {idle }}$.

Assume the current contention window size for node $s$ is $\mathrm{CW}_{s}$, then

$P_{\text {transmit }}=\frac{1}{\text { backoff_timer_value }}=\frac{2}{\mathrm{CW}_{s}}$,

since the value of the backoff timer is a random number in the range $\left[0, C W_{s}\right)$. The probability that the neighbours of the destination node $t$ remain silent is

$P_{\text {idle }}=P_{\text {silent }}^{\text {(number_of_active_neighbours) }}$,

where the number of active neighbours is given by $D \times \pi \times R_{\max }^{2} \times P_{\text {active. }}$ In order to calculate the probability for neighbours to remain silent, we assume that the contention window size for neighbours of $s$ is, on average, $\mathrm{CW}_{\mathrm{avg}}$. The average backoff timer value is $C W_{\text {avg }} / 2$, therefore $P_{\text {silent }}=$ $1-\frac{2}{\mathrm{CW}_{\text {avg }}}$.

This allows Eq. (1) to be rewritten as

$P_{\text {success }}=\frac{2}{\mathrm{CW}_{s}} \times\left(1-\frac{2}{\mathrm{CW}_{\mathrm{avg}}}\right)^{D \pi R_{\max }^{2} P_{\text {active }}}$.

Next, we consider the case where a spatial backoff strategy is employed. Eqs. (1) and (2) still hold. Let us assume that the current transmission range of the source node $s$ is $R_{s}$. Now, three conditions must be satisfied:

(i) The source node $s$ is transmitting in the current transmission frame.

(ii) For each transmission power level $i$, each node within a distance of $R_{i}$ of the destination node $t$ must refrain from transmission if its current transmission range is greater than or equal to $R_{i}$.

(iii) For each transmission power level $i$, where $R_{i}>R_{s}$, each node in the area Ring $_{i}$ of node

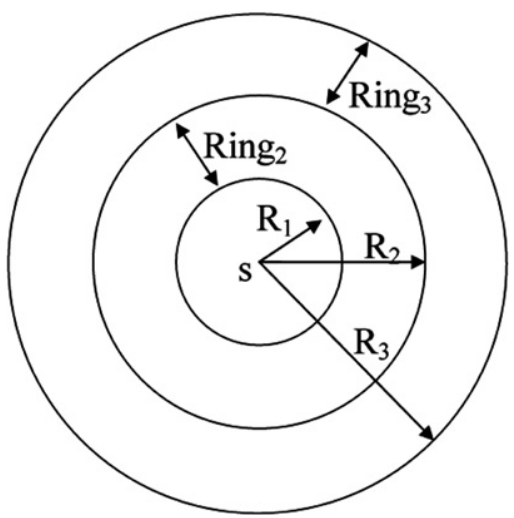

Fig. 25. Rings of node $s$ (located at the center).

$s$ must refrain from transmission for the duration of the transmission if its transmission range is greater than or equal to $R_{i}$. Here, Ring $_{i}$ is the area at distance greater than $R_{i-1}$ and less than or equal to $R_{i}$. Fig. 25 shows Ring $_{i}$ for $i=2,3$. Ring $_{1}=R_{1}$.

The first two conditions ensure that the data packet transmitted by $s$ is received by $t$ correctly. The third condition is used to handle the hidden terminal problem that can arise when variable-range transmission power levels are used (recall Fig. 1 and the associated discussion). The receipt of an ACK at node $s$ may suffer interference from a high power transmission outside of the range of $s$. We must ensure that all nodes in the area described in condition (3) remain silent.

Similar to Eq. (3), the probability that the second condition holds is

$P_{\text {condition } 2}=P_{\text {silent }}^{D \pi P_{\text {active }}} \sum_{i=1}^{n} R_{i}^{2} P_{R_{i}}$,

where $P_{R_{i}}$ is the probability of a node using a transmission range of $R_{i}$, and $n$ is the number of transmission ranges available.

For each ring $\operatorname{Ring}_{i}$, the probability for all nodes in Ring $_{i}$ to refrain from transmission if their transmission range is greater than or equal to $R_{i}$, denoted $P_{\text {idle, } \text { Ring }_{i}}$, is

$P_{\text {idle, } \operatorname{Ring}_{i}}=P_{\text {silent }}^{D \pi\left(R_{i}^{2}-R_{i-1}^{2}\right) P_{\text {active }} P_{\geqslant R_{i}},}$

where $P_{\geqslant R_{i}}$ is the probability of a node using a transmission range greater than or equal to $R_{i}$. This can be written as $P_{\geqslant R_{i}}=\sum_{j=i}^{n} P_{R_{j}}$. 
Combining Eqs. (1), (2), (5), and (6), we have

$$
\begin{aligned}
P_{\text {success }}= & \frac{2}{\mathrm{CW}_{s}} \times\left(1-\frac{2}{\mathrm{CW}_{\mathrm{avg}}}\right)^{D \pi P_{\text {active }} \sum_{i=1}^{b} R_{i}^{2} P_{R_{i}}} \\
& \times \prod_{i, R_{i}>R_{s}}\left(1-\frac{2}{\mathrm{CW}_{\mathrm{avg}}}\right)^{D \pi\left(R_{i}^{2}-R_{i-1}^{2}\right) P_{\text {active }} P \geqslant R_{i}} .
\end{aligned}
$$

Rearranging this equation we obtain

$P_{\text {success }}=\frac{2}{\mathrm{CW}_{s}} \times\left(1-\frac{2}{\mathrm{CW}_{\mathrm{avg}}}\right)^{D \pi P_{\text {active }} E}$,

where the exponential factor $E$ is $\sum_{i=1}^{n} R_{i}^{2} P_{R_{i}}+$ $\sum_{i, R_{i}>R_{s}}\left(R_{i}^{2}-R_{i-1}^{2}\right) \sum_{j=i}^{n} P_{R_{j}}$. The second term of $E$ can be rewritten as $\sum_{i, R_{i}>R_{s}}\left(R_{i}^{2}-R_{s}^{2}\right) P_{R_{i}}$. This allows the expression for $E$ to be simplified as

$E=\sum_{i=1}^{n} R_{i}^{2} P_{R_{i}}+\sum_{i, R_{i}>R_{s}}\left(R_{i}^{2}-R_{s}^{2}\right) P_{R_{i}}$.

\subsection{Discussion}

The probability of successful transmission by a node using temporal backoff and spatial backoff is given by Eqs. (4) and (8), respectively. In both cases $P_{\text {active }}$ and $D$ represent, to some extent, the magnitude of contention in the network. If no backoff strategy is used, i.e., all contention window sizes and transmission ranges remain constant, the probability of success decreases as $P_{\text {active }}$ and $D$ increase. For temporal backoff, increasing the first term $\mathrm{CW}_{s}$ decreases the first part of Eq. (4). However, when

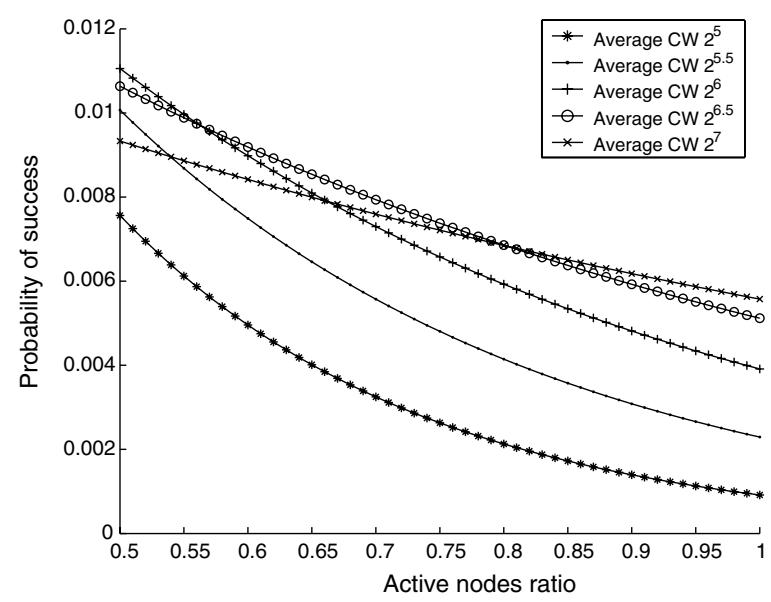

Fig. 26. Probability of success using temporal backoff varying the ratio of active nodes.

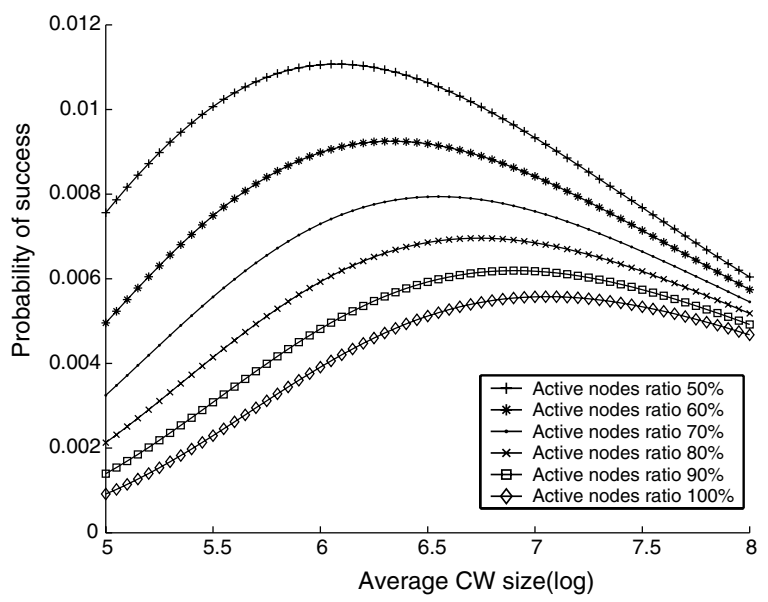

Fig. 27. Probability of success using temporal backoff varying contention window size.

$\mathrm{CW}_{s}$ increases, $\mathrm{CW}_{\mathrm{avg}}$ for other nodes also increases, which increases the second part of Eq. (4).

Figs. 26 and 27 plot the probability of success using temporal backoff under different active node ratios and contention window sizes, respectively. Here, we assume a density $D$ of 50 nodes in an area of $500 \times 300 \mathrm{~m}$ and a transmission range of 250 meters for all nodes. As the active node ratio increases, the probability of a successful transmission decreases because there is more contention in the network. The probability of a successful transmission rises as the contention window size increases when the $\mathrm{CW}$ size is low, and then drops when the CW size is high. This is because when the contention window size is high, a node transmits less frequently. There is an optimal $\mathrm{CW}$ window size for each active node ratio. For an active node ratio of $50 \%$, this value is around $2^{6}$. When the active node ratio is $100 \%$, it increases to around $2^{7.2}$. Although we assume node density is constant, we can interchange the active node ratio with the node density in Figs. 26 and 27. What really affects the results is the product of these two factors.

For a spatial backoff strategy, assuming the CW size is constant, the probability of successful transmission depends solely on the value of the exponential factor $E$ in Eq. (8). From Eq. (9), $E$ is a function of the distribution of transmission power in the network, and the current transmission power. Consider a network with the same density used in temporal backoff. Assume there are three transmission power levels with transmission ranges corresponding to 250, 100, and $40 \mathrm{~m}$. Consider three distributions of transmission power levels: a high, medium, and 
low power scenario where $80 \%$ of the nodes use high, medium, or low transmission power, respectively, with $10 \%$ at each of the two remaining transmission power levels. In all three scenarios, the contention window size is fixed at 32. Figs. 28-30 show the probability of successful transmission given each of these transmission power distributions.

As expected, the probability of a successful transmission decreases as the active node ratio increases. In all three cases, the nodes transmitting at a high transmission power level achieve a high probability of success. This is because these nodes can be hidden to nodes transmitting at low transmission power

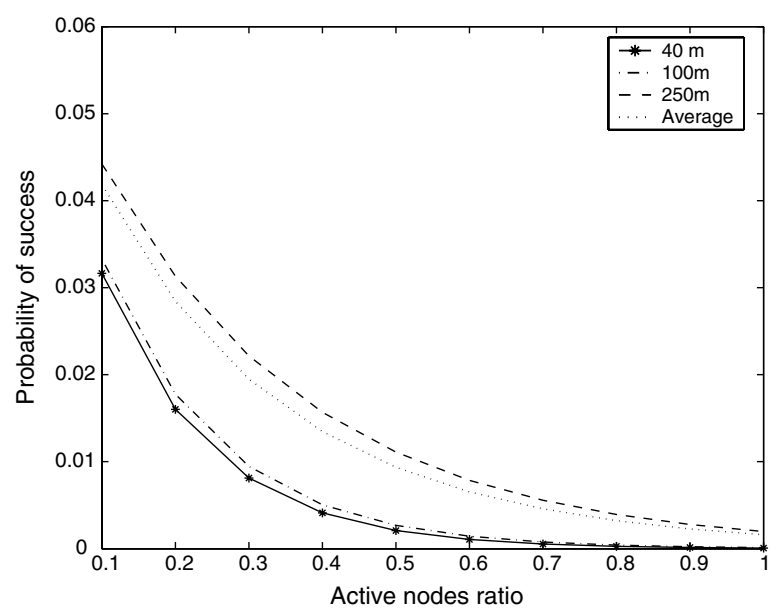

Fig. 28. Probability of successful transmission using spatial backoff as a function of the active node ratio; high power scenario.

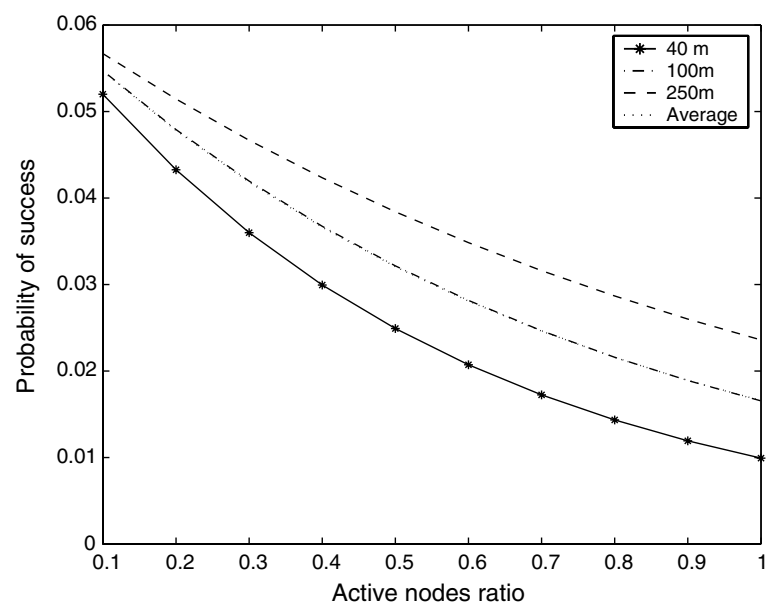

Fig. 29. Probability of successful transmission using spatial backoff as a function of the active node ratio; medium power scenario.

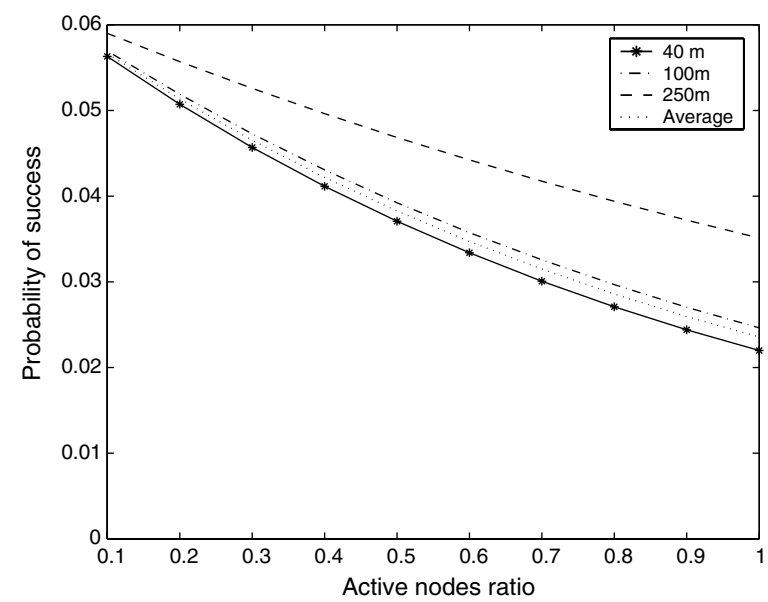

Fig. 30. Probability of successful transmission in spatial backoff as a function of the active node ratio; low power scenario.

levels. The average probability of successful transmission increases as the percentage of nodes transmitting at low transmission power level increases. When the active node ratio is $100 \%$, the average probability of success for the low power scenario is around 10 times higher than the high power scenario. The results of the analysis confirm the potential of space as a means to improve the probability of successful transmission.

\section{Conclusions}

In this paper, we propose an alternate approach to collision resolution in a CSMA protocol, namely the use of power control to resolve collisions by backing off in space. In order to improve performance of the basic protocol under dense or active network conditions, we combine our power backoff (PB) approach in a CSMA protocol, CSMA/PB, with temporal backoff. Simulation results for a variety of static and mobile mobile ad hoc network scenarios show that CSMA/PB always outperforms IEEE 802.11 in throughput per unit energy, often by a significant margin. Analysis confirms the potential of exploiting the dimension of space in medium access control.

We caution that these strong results are based on a centralized power-aware routing strategy that illustrates the potential of power backoff. This suggests that an investigation of CSMA/PB with a distributed power-aware routing protocol is warranted. While asymmetric links seem undesirable, the mobile scenario seems able to take advantage of them. More 
investigation is needed to understand when and how asymmetry can be helpful.

\section{Disclaimer}

The views and conclusions contained in this document are those of the authors and should not be interpreted as representing the official policies, either expressed or implied, of the Army Research Laboratory or the US Government or the National Science Foundation.

\section{Acknowledgements}

We are grateful to the anonymous referees for their careful reading of and helpful comments for our paper. The research of V.R. Syrotiuk is supported, in part, by NSF ANI-0240524 and ITR0220001.

\section{References}

[1] J.T. Gomez, A. Campbell, A case for variable-range transmission power control in wireless multihop networks, in: Proceedings of the 23rd Annual Joint Conference of the IEEE Computer and Communications Societies (Infocom'04), vol. 2, 2004, pp. 1425-1436.

[2] J. Gomez, A.T. Campbell, A case for variable-range transmission power control in wireless multihop networks, IEEE Transactions on Mobile Computing 6 (1) (2007) 87-99.

[3] D. Aldous, Ultimate stability of exponential backoff protocol for acknowledgment based transmission control of random access communication channels, IEEE Transactions on Information Theory 33 (2) (1987) 219-223.

[4] F.P. Kelly, Stochastic models of computer communication systems, Journal of the Royal Statistical Society (B) 47 (1985) 379-395.

[5] J. Hastad, F.T. Leighton, B. Rogoff, Analysis of backoff protocols for multiple access channels, in: Proceedings of the ACM Symposium on the Theory of Computing, 1987, pp. 241-253.

[6] A. Nasipuri, J. Zhuang, S.R. Das, A multichannel CSMA MAC protocol for multihop wireless networks, in: Proceedings of IEEE Wireless Communications and Networking Conference (WCNC'99), 1999, pp. 1402-1406.

[7] A. Nasipuri, S.R. Das, Multichannel CSMA with single power-based channel selection for multihop wireless networks, in: Proceedings of IEEE Vehicular Technology Conference (VTC'00), 2000, pp. 211-218.

[8] J. So, N.H. Vaidya, Multi-channel MAC for ad hoc networks: handling multi-channel hidden terminals using a single transceiver, in: Proceedings of ACM International Symposium on Mobile Ad Hoc Networking and Computing (MobiHoc'04), 2004, pp. 222-233.

[9] Z. Tang, J.J. Garcia-Luna-Aceves, Hop-reservation multiple access (HRMA) for ad-hoc networks, in: Proceedings of the 18th Annual Joint Conference of the IEEE Computer and Communications Societies (Infocom'99), 1999, pp. 194-201.
[10] A. Tzamaloukas, J.J. Garcia-Luna-Aceves, A receiver-initiated collision-avoidance protocol for multi-channel networks, in: Proceedings of the 20th Annual Joint Conference of the IEEE Computer and Communications Societies (Infocom'01), 2001, pp. 189-198.

[11] P. Karn, MACA - a new channel access method for packet radio, in: Proceedings of the Ninth ARRL/CRRL Amateur Radio Computer Networking Conference, 1990, pp. 134 140.

[12] E.-S. Jung, N.H. Vaidya, A power control MAC protocol for ad hoc networks, in: Proceedings of the Eighth ACM International conference on Mobile Computing and Networking (Mobicom'02), 2002, pp. 36-47.

[13] J.P. Monks, V. Bharghavan, W.-M. Hwu, A power controlled multiple access protocol for wireless packet networks, in: Proceedings of the 20th Annual Joint Conference of the IEEE Computer and Communications Societies (Infocom'01), 2001, pp. 219-228.

[14] A. Muqattash, M. Krunz, Power controlled dual channel (PCDC) medium access protocol for wireless ad hoc networks, in: Proceedings of the 22nd Annual Joint Conference of the IEEE Computer and Communications Societies (Infocom'03), 2003, pp. 470-480.

[15] A. Muqattash, M. Krunz, A single-channel solution for transmission power control in wireless ad hoc networks, in: Proceedings of the Fifth ACM International Symposium on Mobile Ad Hoc Networking and Computing (MobiHoc'04), 2004, pp. 210-221.

[16] S. Wu, Y. Tseng, J. Sheu, Intelligent medium access for mobile ad hoc networks with busy tones and power control, IEEE Journal on Selected Area on Communications 18 (9) (2000) 1647-1657.

[17] J. Fuemmeler, N.H. Vaidya, V.V. Veeravalli, Selecting Transmit Powers and Carrier Sense Thresholds for CSMA Protocols, Technical Report, University of Illinois, UrbanaChampaign, October 2004.

[18] B. Chu, Improving IEEE 802.11 Performance with Power Control and Distance Based Contention Window Selection, Master's Thesis, University of Illinois, Urbana-Champaign, 2005.

[19] X. Yang, N.H. Vaidya, On physical carrier sensing in wireless ad hoc networks, in: Proceedings of the 24th Annual Joint conference of the IEEE Computer and Communications Societies (Infocom'05), vol. 4, 2005, pp. 2525-2535.

[20] J. Gomez, A.T. Campbell, M. Naghshineh, C. Bisdikian, PARO: supporting dynamic power controlled routing in wireless ad hoc networks, Wireless Networks 9 (5) (2003) $443-460$

[21] S. Narayanaswamy, V. Kawadia, R.S. Sreenivas, P.R. Kumar, Power control in ad-hoc networks: theory, architecture, algorithm and implementation of the COMPOW protocol, in: Proceedings of the European Wireless Conference, 2002, pp. 156-162.

[22] V. Kawadia, P.R. Kumar, Power control and clustering in ad hoc networks, in: Proceedings of the 22nd Annual Joint conference of the IEEE Computer and Communications Societies (Infocom'03), 2003, pp. 459-469.

[23] K.K. Vadde, V.R. Syrotiuk, Factor interaction on service delivery in mobile ad hoc networks, IEEE Journal on Selected Areas on Communciations 22 (7) (2004) 1335-1346.

[24] K.K. Vadde, V.V. Syrotiuk, D.C. Montgomery, Optimizing protocol interaction using response surface methodology, 
IEEE Transactions on Mobile Computing 5 (6) (2006) 627 639.

[25] The network simulator - ns-2, University of California, Berkeley. http://www.isi.edu/nsname/ns/.

[26] M. Cesana, D. Maniezzo, P. Bergamo, M. Gerla, Interference aware (IA) MAC: an enhancement to IEEE $802.11 \mathrm{~b}$ DCF, in: Proceedings of the IEEE Vehicular Technology Conference (VTC-Fall'03), vol. 5, 2003, pp. 2799-2803.

[27] T. Kim, J.C. Hou, H. Lim, Improving spatial reuse through tuning transmit power, carrier sense threshold, and data rate in multihop wireless networks, in: Proceedings of the 12th Annual International conference on Mobile Computing and Networking (Mobicom'06), 2006, pp. 366-377.

[28] Q. Chen, D. Jiang, V. Taliwal, L. Delgrossi, IEEE 802.11 based vehicular communication simulation design for ns-2, in: Proceedings of the Third International Workshop on Vehicular Ad Hoc Networks (VANET'06), 2006, pp. 50-56.

[29] W. Navidi, T.K. Camp, Stationary distributions for the random waypoint mobility model, IEEE Transactions on Mobile Computing 3 (1) (2004) 99-108.

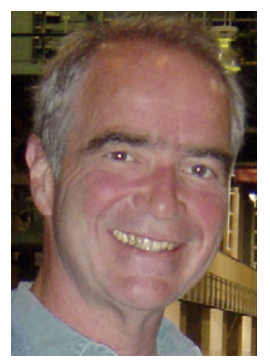

Charles J. Colbourn earned his M.Math. in 1978 from the University of Waterloo, and his Ph.D. in 1980 from the University of Toronto, both in Computer Science. He has held academic positions at the University of Saskatchewan, the University of Waterloo, the University of Vermont, and is currently a Professor of Computer Science and Engineering at Arizona State University. Dr. Colbourn is the author of The Combinatorics of Network Reliability (Oxford) and Triple Systems (Oxford). He is editor-in-chief of the Journal of Combinatorial Designs and serves on the editorial boards of Networks, Discrete Mathematics, Journal of Combinatorial Theory (A), Designs, Codes and Cryptography, and others. He edited the standard reference work The CRC Handbook of Combinatorial Designs. He is the author of more than 250 refereed journal papers focussing on combinatorial designs and graphs with applications in networking, computing, and communications. In 2004, he was awarded the Euler Medal for Lifetime Research Achievement by the Institute for Combinatorics and its Applications.

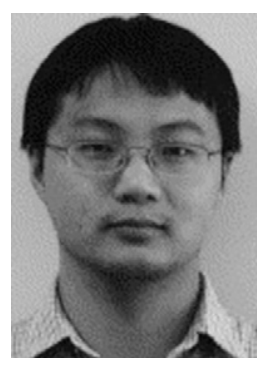

Minghao Cui received his B.E. in Computer Science and Engineering from Harbin Institute of Technology, China, in 2002, and M.S. in Computer Science and Engineering from Arizona State University, in 2004. He is currently a Ph.D. student in Computer Science and Engineering at Arizona State University. His research interests include mobile ad hoc networks and computing, medium access control protocols with an emphasis on transmit power control and cooperative transmission.

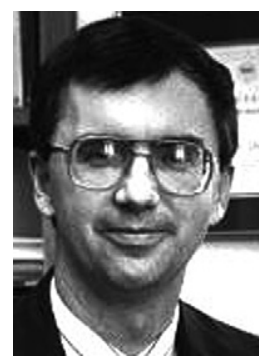

Errol Lloyd is a Professor of Computer and Information Sciences at the University of Delaware. Previously he served as a faculty member at the University of Pittsburgh and as Program Director for Computer and Computation Theory at the National Science Foundation. From 1994 to 1999 he was Chair of the Department of Computer and Information Sciences at the University of Delaware. Concurrently, from 1997 to 1999 he was Interim Director of the University of Delaware Center for Applied Science and Engineering in Rehabilitation. Professor Lloyd received undergraduate degrees in both Computer Science and Mathematics from Penn State University, and a Ph.D. in Computer Science from the Massachusetts Institute of Technology. His research expertise is in the design and analysis of algorithms, with a particular concentration on network algorithms. In 1989 Professor Lloyd received an NSF Outstanding Performance Award, and in 1994 he received the University of Delaware Faculty Excellence in Teaching Award.

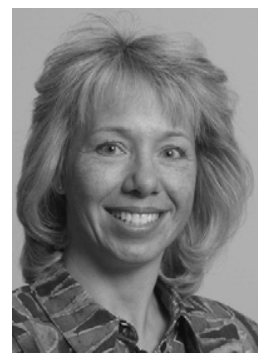

Violet R. Syrotiuk (M'96/ACM'93) earned her Ph.D. in Computer Science from the University of Waterloo (Canada) in 1992. She joined Arizona State University in 2002 and is currently an Associate Professor of Computer Science and Engineering. Dr. Syrotiuk's research is currently supported by two grants from NSF, and contracts with Los Alamos National Laboratory and Defence Science and Technology Organisation (Australia). She serves on the Editorial Board of Computer Networks, and on the Technical Program Committee of several major conferences including Mobicom, Mobihoc, and Infocom. Her research interests include MAC and higher layer protocols for multi-hop wireless networks. 